\title{
Aplicando Transparência no Domínio de Informação Sobre Saúde na Internet: Uma Abordagem Visando o Cidadão
}

\author{
Nathália Miranda do Nascimento ${ }^{1}$, Henrique Prado de Sá Sousa ${ }^{1}$, Eduardo Kinder \\ Almentero', Julio Cesar Sampaio do Prado Leite ${ }^{2}$ \\ ${ }^{1}$ Departamento de Computação (DECOMP) - Universidade Federal Rural do Rio de \\ Janeiro (UFRRJ) - Seropédica - RJ - Brasil \\ nathalia_miranda93@yahoo.com.br, \{hsousa, almentero\}@ufrrj.br
}

${ }^{2}$ Departamento de Informática - Pontifícia Universidade Católica do Rio de Janeiro (PUC-Rio) - Rio de Janeiro - RJ - Brasil

juliodinf.puc-rio.br

\begin{abstract}
The internet has renewed the way communication and access to health information are made. However, the Internet has some difficulties related to the quality and access of health information. Transparency is a current demand of society, and the provision of more transparent health information by means of the internet demands special attention to its quality. The problem addressed in this work is related to the search and access to good quality health information available on the internet. To address this issue, an approach based on identification, selection and consolidation of information has been proposed. This approach culminated in the development of a prototype, which was used in a preliminary evaluation, the results of which are described.
\end{abstract}

Resumo. A internet renovou a forma como a comunicação e o acesso às informações de saúde são feitos. Porém, a internet está sujeita a dificuldades referentes à busca e qualidade da informação sobre saúde. A Transparência é uma demanda atual da sociedade, e o fornecimento de informações de saúde mais transparentes vai de encontro às tecnologias como a internet como um item de qualidade. O problema abordado neste trabalho está relacionado com a busca e com o acesso a informação de boa qualidade em saúde disponível na internet. Afim de tratar esta questão, uma abordagem baseada na identificação, seleção e consolidação de informações foi proposta. Tal abordagem culminou no desenvolvimento de um protótipo, que foi utilizado em uma avaliação preliminar, cujos resultados são descritos.

\section{Introdução}

Pesquisas mostram que nos últimos anos o uso das TICs (Tecnologias de Informação e Comunicação) se intensificou e o processo de difusão da Internet se massificou, resultando no aumento da produção e propagação da informação sobre variados assuntos e em diferentes formatos. Entretanto, as informações disponibilizadas na internet não possuem garantia de qualidade e sequer de veracidade (MENDONÇA, 2013), não obstante, são frequentemente utilizadas para empregar falsas verdades (SIMÕES, 2016). Além disso, o grande volume de informações dificulta a localização de uma fonte confiável (TOMAÉL\&VALENTIM, 2004). 
Essa questão se torna especialmente relevante no contexto em que a internet passa a ser utilizada como uma ferramenta para busca de conhecimento sobre saúde, não raro, substituindo consultas médicas (COELHO et al., 2013). Dados de 2012 indicam que 43\% dos usuários de Internet buscaram informação relacionada à saúde ou aos serviços de saúde, representando a terceira maior atividade dos brasileiros na busca por informação online (BARBOSA, 2013), e pesquisas nacionais e internacionais indicam que o número de pessoas que utilizam a Internet para obter informações sobre doenças, tratamentos e até mesmo diagnósticos tende a aumentar cada vez mais (MORETTI et al., 2012, AMANTE et al., 2015). Logo, o acesso à informação de qualidade é parte integrante da promoção da saúde, que visa maior controle e poder de decisão ao usuário (GARBIN et al., 2012).

No entanto, existe grande número de sites sobre temas de saúde-doença, bemestar, qualidade de vida e promoção da saúde, produzidos por organizações ou indivíduos sem qualquer tipo de avaliação (PAOLUCCI, 2014). Muitos apresentam informações contraditórias, incorretas, incompreensíveis ou até fraudulentas. Há um consenso entre os autores sobre o risco apresentado pela busca de informação em saúde na Internet (MENDONÇA, 2013). Desta forma, a qualidade da informação tem se transformado em um novo desafio para a Saúde Pública no mundo (FIOCRUZ, 2016).

As buscas nas ferramentas tradicionais (ex. Google, Yahoo e Bing) não diferenciam as informações presentes nos sites em termos de qualidade, o que pode contribuir, por exemplo, ao acesso de informações dúbias, inconsistentes e sem garantia de veracidade. Entretanto, são as ferramentas mais utilizadas pela maioria das pessoas (independente de gênero e faixa etária), em contrapartida aos sites especializados, portais de notícia e fóruns (MINHAVIDA, 2016).

A partir das deficiências relacionadas a qualidade de informação na internet, mais especificamente no domínio da saúde, buscamos propor operacionalizações que facilitem o acesso a informações mais confiáveis e completas. No primeiro passo, avaliamos o conteúdo de diversos sites de saúde e identificamos as propostas existentes na área (Saúde) para reduzir as dificuldades de confiabilidade da informação. Este estudo culminou na definição de operacionalizações, as quais foram consolidadas em um processo que visa ampliar qualidade da informação de saúde na internet.

Tal processo foi baseado no uso de critérios para seleção de um conjunto de fontes de informação, o qual foi utilizado para a construção de um modelo conceitual consolidado, que subsidiou a criação de um protótipo de portal. Na construção do portal foram considerados alguns quesitos de Transparência (GER-PUC, 2018) para a operacionalização de elementos de interface. Posteriormente o portal foi avaliado por potenciais usuários, em comparação aos meios de busca tradicionais. Os resultados são resumidos neste artigo.

\section{Processo Proposto}

Os principais problemas identificados na busca por informações de saúde na internet estão relacionados à credibilidade das informações presentes em sites e aos diferentes focos dado ao tema que criam dispersão de informações sobre o assunto buscado. Além disso, alguns sites se aprofundam mais e outros menos em explicações sobre os determinados assuntos. 
A escolha das fontes de informação que devem ser utilizadas em uma busca por dados sensíveis como os de saúde devem seguir critérios que mantenham algum grau mínimo de confiança acerca dos dados que ela disponibiliza. Um dos passos que realizamos foi a definição de critérios para a seleção das fontes de informação. Identificamos que existem certificados emitidos por órgãos de saúde a determinados sites que se encontram em conformidade com critérios específicos de qualidade da área (ex. (HON, 1999), (FIOCRUZ, 2018). Outros critérios definidos foram a aceitação de informações provenientes de sites governamentais, instituições de saúde e universidades.

Partindo dos critérios, selecionamos algumas fontes de informação para aplicação neste estudo. Posteriormente mapeamos os conceitos oferecidos pelas fontes de informação. Esses modelos serviram de insumo para a construção de um modelo consolidado, que contém todas as informações disponibilizadas nas diferentes fontes. $\mathrm{Na}$ atividade de consolidação, foram avaliados os sinônimos para que não houvesse repetição de conceitos. $\mathrm{O}$ modelo consolidado foi utilizado como requisito para a definição de um protótipo com a capacidade de apresentar essas informações de forma integrada, permitindo ao usuário acessar o conteúdo das fontes de informação selecionadas através de uma busca facilitada. Posteriormente foi definido um protótipo que propõem uma forma de busca e apresentação dessas informações.

As atividades propostas criam um fluxo de identificação, seleção e apresentação de informações (Figura 1).

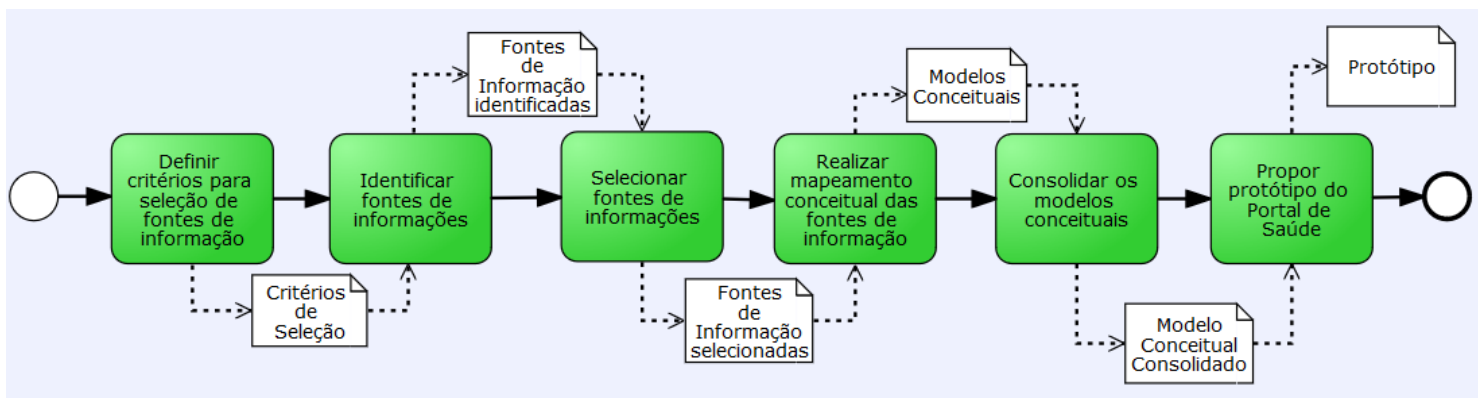

Figura 1 - Abordagem para ampliar qualidade da informação de saúde na internet

Para a definição do protótipo, algumas qualidades foram consideradas para apresentar as informações com maior grau de Transparência. Para isso foi utilizado o Catálogo de Transparência.

\subsection{Identificação e seleção das fontes de informação}

Para a identificação das fontes, foi realizada uma busca através do Google, mediante a busca por palavras-chave. Para simular o comportamento de um usuário comum, que busca informação na internet e desconhece a possibilidade do uso do vocabulário controlado e estratégias de buscas, optou-se por descritores de pesquisa escritos em português, sendo utilizados em várias combinações: portal de saúde; site de doença, site de saúde.

Na busca avançada os limites estabelecidos foram no modo "Pesquisa avançada", opção "páginas em português" e país "Brasil", e foram designados para busca avançada os mesmos descritores da busca simples em português mais a string de busca (("saúde") AND ("site" OR "portal") AND ("doença")), usando recurso de marcadores para 
delimitar a pesquisa, tais como, as aspas e o sinal de positivo que servem como filtros na pesquisa avançada.

Após a identificação, foi obtida uma amostra de trinta e seis sites. A partir desta amostra, foi realizada a seleção de sites considerando os critérios de inclusão estabelecidos, que são:

Critérios de inclusão:

- Websites que tenham acreditação ou certificação da qualidade da informação na internet;

- Websites governamentais;

- Websites de instituições médicas;

- Websites que estejam entre os resultados apresentados até a página 3 do Google;

- Websites que contenham informações sobre doenças, por exemplo, sintomas e tratamento.

Entre os sites que foram retornados pelo sistema de busca e que não atendem aos critérios estabelecidos, temos como exemplo:

- Websites pessoais (blogs);

- Websites de teses e dissertações;

- Websites de ofertas de cursos referentes a temática;

- Website que abordam um único assunto;

Ao fim da seleção, os seguintes (Tabela 1) sites foram selecionados:

Tabela 1 - Lista dos Portais de Saúde Selecionados

\begin{tabular}{|l|l|}
\hline \multicolumn{1}{|c|}{ Nome } & \multicolumn{1}{c|}{ Endereço } \\
\hline $\begin{array}{l}\text { Portal sobre doenças raras e medicamentos } \\
\text { órfãos - Orphanet }\end{array}$ & $\begin{array}{l}\text { http://www.orpha.net/consor/cgi- } \\
\text { bin/index.php?lng=PT }\end{array}$ \\
\hline Minha Vida & http://www.minhavida.com.br/ \\
\hline DeCS - Descritores em Ciências da Saúde & http://decs.bvs.br/ \\
\hline Jornal Conceito Saúde & $\begin{array}{l}\text { http://www.jornalconceitosaude.com.br/menu- } \\
\text { doencas/ }\end{array}$ \\
\hline Ministério da Saúde - Portal da Saúde & http://portalsaude.saude.gov.br/ \\
\hline Criasaude & https://www.criasaude.com.br/ \\
\hline
\end{tabular}

\subsection{Mapeamento e consolidação das informações}

A partir do conjunto de fontes de informação selecionado, foi feito o mapeamento dos conceitos presentes nos sites de saúde para reunir suas informações e consolidá-las, visando obter um modelo único do conhecimento presente nos sites. Com esse resultado espera-se ampliar o grau de completeza das informações.

A realização do mapeamento de informações consistiu em uma busca no site em seus diferentes níveis de páginas visando, em especial, identificar os conceitos chaves. Os recursos que mais forneceram informações foram os nomes de itens dos menus e os títulos internos das páginas. O mapeamento de cada site auxiliou a tornar o seu conteúdo mais transparente, o que nos permitiu analisar as diferenças nas informações presentes. 
O conhecimento elicitado desses sites foi utilizado para construir modelos conceituais escritos na linguagem UML (Unified Modeling Language). O modelo conceitual gerado representa os conceitos referentes ao domínio do problema abordado. As diferenças de conceitos presentes nos sites (Figura 2 e Figura 3) explicitam a falta de padronização nas informações que são exibidas aos usuários.

Os modelos mostram que os sites abordam diferentes conceitos, e podem possuir mais ou menos detalhamento de informações sobre um mesmo conceito. Isso demonstra que o uso de uma única fonte de informação pode ser insuficiente para fornecer informações abrangentes sobre determinado assunto.

A partir dos modelos de cada portal foi construído um Modelo Conceitual Consolidado. A consolidação consistiu na união dos conceitos presentes nos sites, bem como a consolidação de termos que possuíam diferentes atributos. Basicamente conceitos e seus atributos foram somados ao modelo consolidado, eliminando-se repetições por palavras similares, especialmente entre os atributos. Este modelo não é apresentado aqui por motivos de espaço.

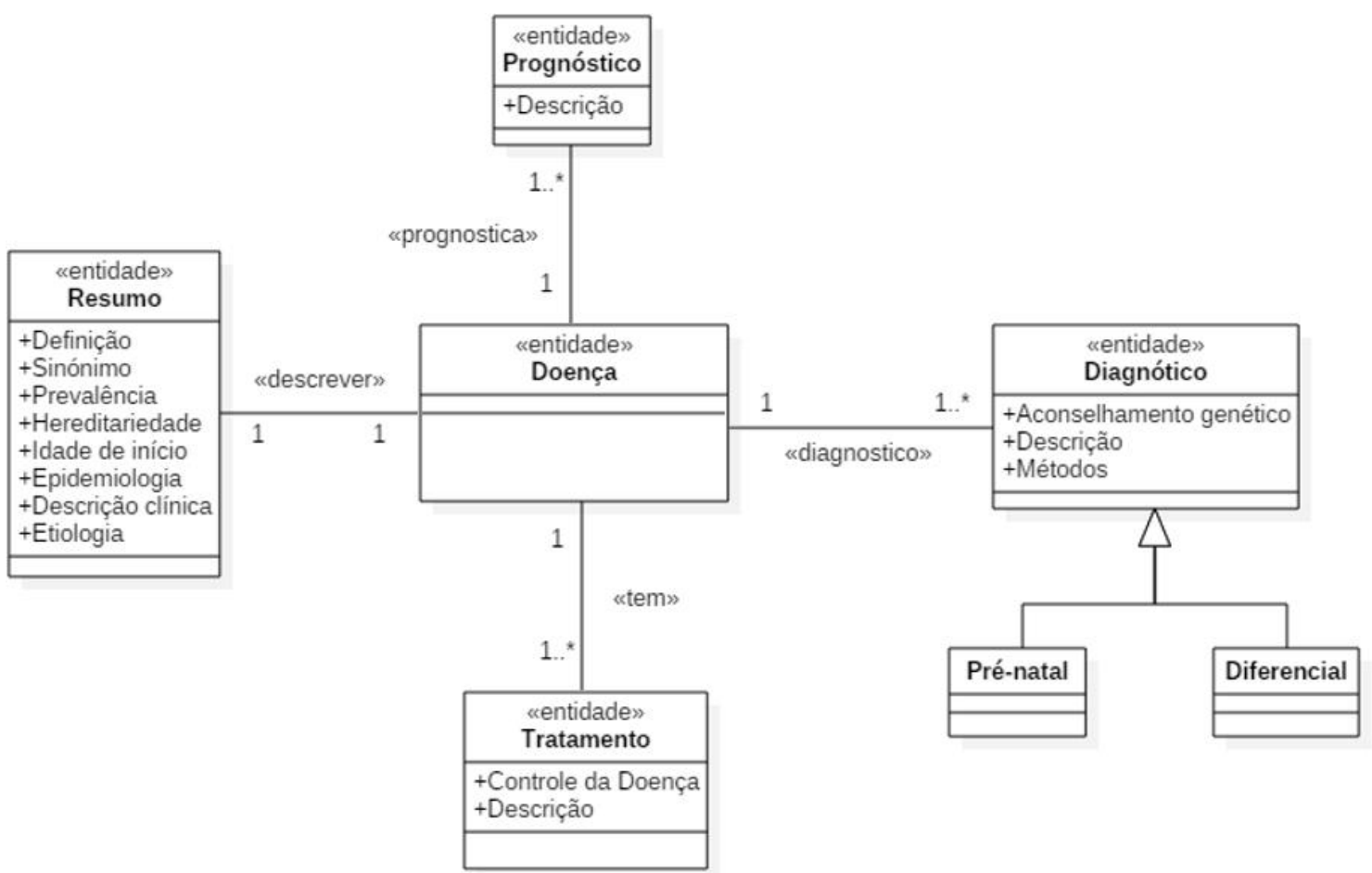

Figura 2 - Exemplo de modelo conceitual do Portal Orphanet 


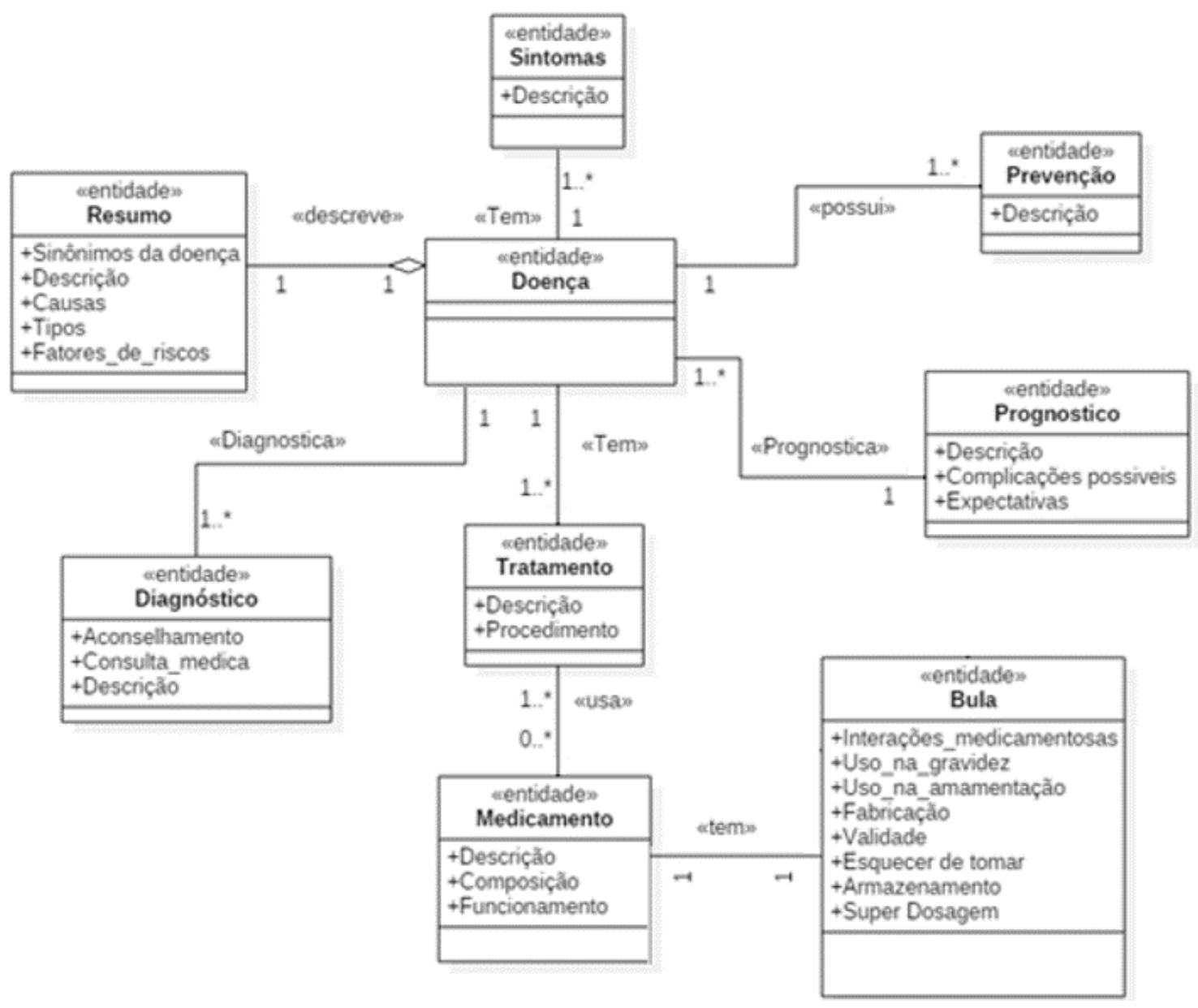

Figura 3 - Exemplo de modelo conceitual do Minha Vida

\subsection{Construção do protótipo}

A projeção do protótipo se baseou em operacionalizações que visam incrementar algumas das qualidades presentes no Catálogo de Transparência (GER-PUC).

O Catálogo de Transparência é fundamentado no GQM (Goal, Question, Metrics) (BASILI, 1992) e se baseia na decomposição de características de qualidade que, por sua vez, são refinadas em grupos de perguntas. As operacionalizações são formas de responder a estas perguntas e é daí que provêm a sua flexibilidade. Existem diversas formas de responder às perguntas existentes no catálogo e, consequentemente, inúmeras operacionalizações que contribuem para a transparência.

Neste trabalho foi feito a instanciação do catálogo de Transparência visando à transparência da informação sobre saúde ao cidadão. Mapeamos de forma básica algumas das contribuições positivas resultantes de atividades presentes na proposta de abordagem para ampliar qualidade da informação de saúde na internet, conforme ilustra a Figura 4. Por exemplo, a atividade "Definir critérios para fontes de informação" contribui tanto para Corretude quanto para Confiabilidade, ambas aplicadas à informação de saúde na internet. 


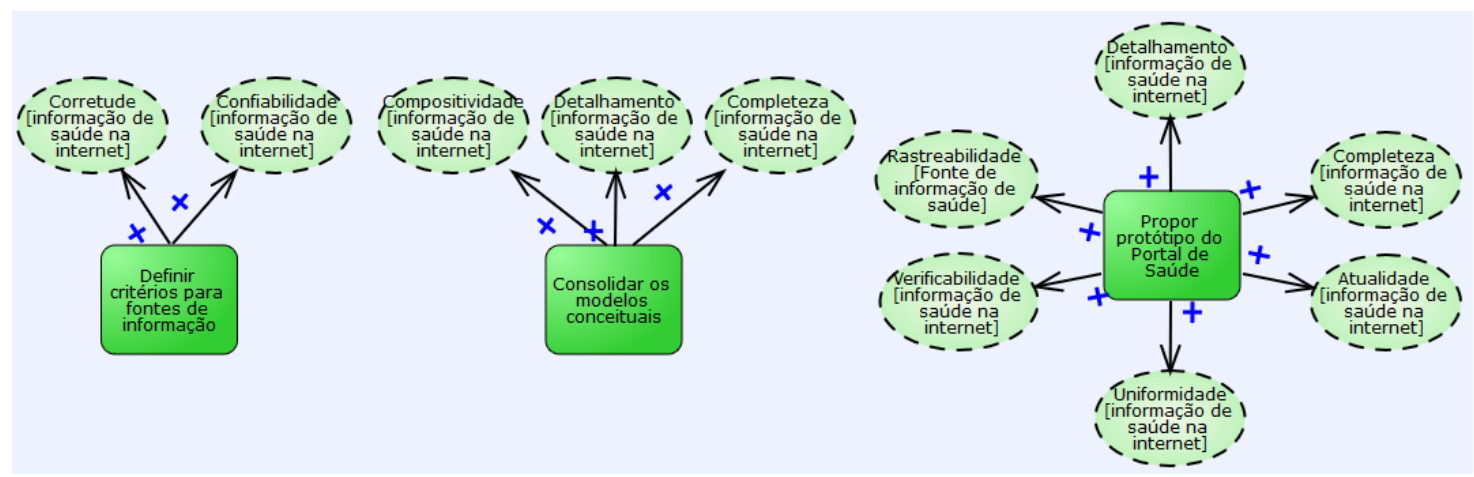

Figura 4 - Contribuições resultantes de atividades presentes na abordagem de transparência da informação de saúde na internet

No protótipo, em especial, foram operacionalizados outros atributos de transparência visando ampliar o grau de qualidade da informação. Por exemplo, para promover algum grau de rastreabilidade com as fontes das informações, foram incluídas as referências e elos para os sites originais, respaldando assim os conteúdos. Isso também ajuda a incrementar a qualidade de Verificabilidade da informação. Outra qualidade que se buscou incrementar foi a Atualidade, operacionalizada pela adição de informações como a data de publicação da informação e data da última atualização.

O grau de Completeza aumenta com a oferta de mais informações sobre diferentes aspectos de determinado assunto, possibilitado pela composição de informações que foi realizada na consolidação das fontes de informação (por consequência, também se alcança maior grau para a qualidade de Detalhamento). Também se contribuiu para a Uniformidade com a padronização na apresentação das informações. A Figura 5Figura 5 apresenta o resultado parcial de uma busca no protótipo do portal de saúde, demonstrando as operacionalizações referenciadas anteriormente. Outras qualidades foram operacionalizadas no protótipo, mas não são apresentadas aqui por restrições de espaço.

\section{Botulismo}

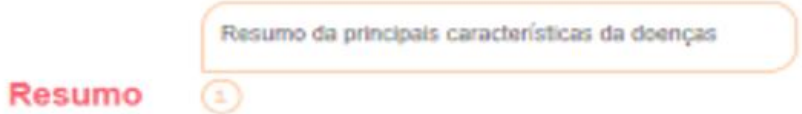

\section{Definiçăo}

Fonte: Descritores em Cièncias da Saúde (DeC S)

$>$ Fonte: Minha Vida

FONTE: ORPHANET

O Botulismo é uma đoença rara da junçäo neuromuscular, adquirida, caracterizada por paralisla flácida

descendente causada por neurotoxinas botulinicas (BoNTs), inclui quatro formas com diferentes modos de

aquisiça.

Fonde: Orpha net 
Nesta primeira versão do protótipo, foram implementadas a simulação de duas buscas por doenças (botulismo e artrose). Ainda não foram implementadas buscas automáticas nos sites mapeados. As buscas simuladas foram utilizadas na avaliação.

\section{Avaliação}

O protótipo foi avaliado por meio de uma pesquisa de opinião com potenciais usuários através de um questionário para verificar se o cidadão identifica as contribuições qualitativas que se buscou ao aplicar as operacionalizações propostas.

O questionário é composto de 25 questões fechadas de múltipla escolha e 1 questão aberta, para que os respondentes explicitem a sua opinião pessoal. Ao todo recebemos 21 questionários respondidos.

Para o preenchimento do questionário foi definido um procedimento de busca para que o usuário pudesse comparar a busca tradicional e a busca no protótipo do portal, e assim tivessem um conhecimento prévio suficiente para poder responder o questionário. Os procedimentos realizados pelos respondentes foram: (1) Fazer uma busca pelas doenças estipuladas (botulismo e artrose) através do Google, livremente, em seguida observar o resultado gerado, selecionar um ou mais sites para acessar e verificar as informações oferecidas por este(s) site(s); (2) Fazer uma busca no portal proposto pelas mesmas doenças estipuladas na etapa anterior. Observar o resultado gerado; (3) Responder ao questionário comparando as duas buscas e seus resultados.

Entre os principais resultados obtidos na comparação entre a busca tradicional e a busca no portal, apresentamos os seguintes resultados:

$61,9 \%$ concordam que há maior facilidade na realização de buscas no portal.

$66,6 \%$ concordam que encontraram informações mais completas no portal. no portal.

$71,4 \%$ concordam que foi possível entender mais sobre o assunto a partir da busca

$76,2 \%$ concordam que as informações apresentadas de diferentes fontes foram úteis para que os respondentes tirassem suas dúvidas.

$76,2 \%$ dos respondentes concordam que tiveram maior confiança na pesquisa porque sabiam que o portal se baseia somente em sites certificados e de instituições governamentais.

Verificou-se que $90,5 \%$ dos respondentes que pesquisaram através do protótipo não utilizaram novas fontes de informação em sua busca. Já dentre os que utilizaram o Google, $47,5 \%$ foram a 2 ou mais sites para tirarem as suas dúvidas, indicando que o portal proporcionou maior eficiência e completeza de informações.

\section{Conclusão}

Este trabalho abordou os problemas relacionados com a busca e qualidade de informações de saúde disponíveis na internet. A fim de tratar este problema, foi proposto um processo que, em suma, proporciona a identificação, mapeamento, consolidação e apresentação de informações (através de um protótipo).

A construção do protótipo para apresentação das informações também considerou características de qualidade presentes no Catálogo de Transparência de Software (GER- 
PUC). Além disto, foi possível verificar que características presentes no catálogo foram influenciadas pelo processo proposto neste trabalho. Por conseguinte, também foi proporcionada uma contribuição no âmbito do catálogo de transparência, uma vez que as ações aqui propostas podem ser incorporadas no catálogo como possíveis operacionalizações de requisitos não funcionais, permitindo seu reuso no futuro.

Também foi realizada uma avaliação com potenciais usuários através do protótipo. A avaliação permitiu, apesar de suas limitações, chegar à conclusão que há indícios de que o trabalho proposto contribui para melhoria da busca e qualidade de informações sobre saúde na internet.

Contudo, é importante destacar que o um dos resultados alcançados é um protótipo, ou seja, não houve de fato a implementação de um software, e sim uma simulação do comportamento. Isto pode ter influenciado a percepção das pessoas que o utilizaram durante o procedimento de avaliação realizado, o que, consequentemente, limita as nossas conclusões. Além disto, outro fator que limita as conclusões é o número de respostas do questionário recebidas.

Como trabalho futuro pretendemos desenvolver o portal, avaliá-lo novamente com um número maior de usuários, estimular a aplicação do modelo proposto, fazer avalição do portal através do catálogo e usar o catálogo para avaliar outros sites. Além disso, intencionamos utilizar o processo proposto em outros domínios e, assim, avaliar a sua contribuição para um contexto de transparência de informação mais amplo.

\section{Referências}

AMANTE, D. J., HOGAN, T. P., PAGOTO, S. L., ENGLISH, T. M., LAPANE, K. L., Access to care and use of the Internet to search for health information: results from the US National Health Interview Survey. Journal of medical Internet Research, v. 17, n. 4, 2015.

BARBOSA, A. Pesquisa sobre o uso das tecnologias de informação e comunicação no Brasil: TIC Domicílios e TIC Empresas 2011. São Paulo: Comitê Gestor da Internet no Brasil, 2013.

BASILI, V.R., Software modeling and measurement: the Goal/Question/Metric paradigm, 1992.

COELHO, E.Q., COELHO, A.Q., CARDOSO, J.E.D., Informações médicas na internet afetam a relação médico-paciente?, Revista Bioética, 21(1), pp.142-149, 2013.

FIOCRUZ - Fundação Oswaldo Cruz Inovação em Saúde para a sociedade. Selo de Qualidade da Informação em Saúde na Internet. 2017. Disponível em: < http://portfolioinovacao.fiocruz.br/?page_id=1450>. Acessado em: 20/03/2017.

GARBIN, H.B.R., GUILAM, M.C.R., NETO, A.F.P., Internet na promoção da saúde: um instrumento para o desenvolvimento de habilidades pessoais e sociais. Physis-Revista de Saúde Coletiva, v. 22, n. 1, 2012.

GER-PUC (Grupo de Engenharia de Requisitos da PUC-Rio), Catálogo Transparência, $2017 . \quad$ Disponível em: $\quad<\mathrm{http} / /$ transparencia.inf.pucrio.br/wiki/index.php/Cat\%C3\%A1logo_Transpar\%C3\%AAncia>. Acessado em: 11 março. 2018. 
HON - Hon Foundation. Código de conduta para sites web em Medicina e Saúde (HONcode), 1999.

MENDONÇA, A.P.B., Critérios de qualidade para sites de saúde: uma proposta, p. 97, Rio de Janeiro: ENSP/Fiocruz, 2013. Disponível em: < http://www6.ensp.fiocruz.br/repositorio/resource/369214>. Acesso em: 10 mai. 2016.

MINHAVIDA, A Jornada digital do paciente, 2016.

MORETTI, F. A., OLIVEIRA, V. E., SILVA, E.M.K., Acesso a informações de saúde na internet: uma questão de saúde pública?, Revista da Associação Médica Brasileira, v. 58, n. 6, p. 650-658, 2012.

PAOLUCCI, R., A necessidade de uma revisão da avaliação da informação online, Mestrado em Informação e Comunicação em Saúde, IES:ICICT/FIOCRUZ. Congresso Brasileiro Interdisciplinar de Promoção da Saúde, 2014.

SIMÕES, A.L., Hoax - o avanço tecnológico e a propagação de boatos, IX Simpósio Nacional ABCiber, PUC-SP, 2016.

TOMAÉL M.I., VALENTIM, M.L.P. Avaliação de fontes de informação na internet. Londrina: Eduel; 2004. 